\title{
Power Quality Improvement Using Fuzzy Logic Control Static Var Compensator In Power System Network
}

\author{
${ }^{1}$ Javid Akhtar, ${ }^{2}$ Shamsudheen.P.M \\ ${ }^{1}$ Associate professor Dept of Electrical and Electronics Engineering, Ghousia College of Engineering, \\ Ramanagaram \\ ${ }^{2}$ M.Tech student of power system engineering Dept of Electrical and Electronics Engineering, Ghousia College \\ of Engineering, Ramanagaram
}

\begin{abstract}
Static Var Compensator (SVC) is a shunt type FACTS which is used in power system primarily for the purpose of voltage and reactive power control in the power system network. In this paper, a fuzzy logic based supplementary controller for Static Var Compensator (SVC) is developed which is used for improve the power quality in generation, transmission and distribution side of power system network. In the generation part of power system fuzzy logic based svc controller is used to damping the rotor angle oscillations and to improve the transient stability of the power system. At the distribution side to minimize the harmonics injected into the distribution systems by the operation of TSC-TCR type SVC used in conjunction with fast changing loads at LV distribution level. Fuzzy logic system and ANN are going to be used solve this nonlinear problem, giving optimum triggering delay angles used to trigger switches in TCR. Fuzzy rules are easily derived from the measurable global signals like line active power flow, and generator speed deviation. The performance of various controllers is then compared based on non linear simulation results. Among these the performance of the proposed controller is found to be better and damp out the system oscillations at faster rate. SVC controller works accurately. Digital computer simulations were performed using MATLAB/ SIMULINK software.
\end{abstract}

Index Terms: - Static Var compensator (SVC) artificial neural network (ANN)

\section{INTRODUCTION}

Power quality is an issue that is becoming increasingly important to electricity consumers at all levels of usage. Sensitive equipment and non-linear loads are commonplace in both the industrial and the domestic environment, because of this a heightened awareness of power quality is developing. The sources of problems that can disturb the power quality are: power electronic devices, arcing devices, load switching, large $\backslash$ motor starting, embedded generation, sensitive equipment, storm and environment related damage, network equipment and design. The solution to improve the energy quality (PQ-Power Quality) at the load side is of great important when the Custom Power; a concept that we could include among the FACTS, but that is different to them because of their final use. In fact the topologies that they employ are identical to the ones in the FACTS devices with little modifications and adaptations to tension levels; therefore they are most oriented to be used in distribution networks of low and medium tension, sometimes replacing the active filters. Flexible AC Transmission Systems (FACTS) devices with a suitable control strategy have the potential to increase the system stability hunt FACTS devices play an important role in reactive power flow in the power network. In large power systems low frequency electro-mechanical oscillations often follow the electrical disturbances. To achieve damping a shunt FACTS device Static Var Compensator (SVC) designed with auxiliary controllers. Therefore SVC is more effective and if accommodated with supplementary controller, by adjusting the equivalent shunt capacitance, SVC will damp out the oscillations and improves the overall system stability. The system operating conditions change considerably during disturbances. Various approaches are available for designing auxiliary controllers in SVC.

An attractive feature of fuzzy logic control is its robustness in system parameters and operating conditions changes. Fuzzy logic controllers are capable of tolerating uncertainty and imprecision to a greater extent.

\section{METHODOLOGY}

\section{A. STATIC Var COMPENSATOR}

This Thesis will commence with an overview of the problems encountered with Shunt compensator (SVC).The Static Var Compensator (SVC) is a shunt type FACTS device which is used to improve the power quality for the purpose of voltage and reactive power control. 


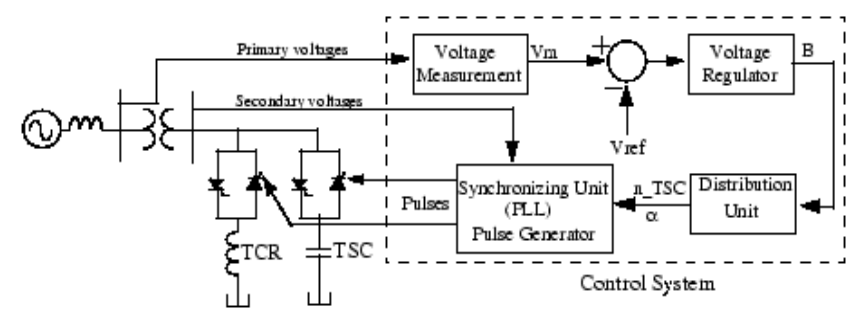

Fig 1 Single line diagram of an SVC and its control system block diagram

The control system consists of

- A measurement system measuring the positive-sequence voltage o be controlled. A Fourier-based measurement system using a one-cycle running average is used.

- A voltage regulator that uses the voltage error (difference between the measured voltage $V_{m}$ and the reference voltage $\mathrm{V}_{\text {ref }}$ ) to determine the SVC susceptance B needed to keep the system voltage constant.

- A distribution unit that determines the TSCs (and eventually TSRs) that must be switched in and out, and computes the firing angle of TCRs.

- A synchronizing system using a phase-locked loop (PLL) synchronized on the secondary voltages and a pulse generator that send appropriate pulses to the thyristors.

The SVC (Phasor Type) block is a phasor model, and you must use it with the phasor simulation method, activated with the Powergui block. It can be used in three-phase power systems together with synchronous generators, motors, and dynamic loads to perform transient stability studies and observe impact of the SVC on electromechanical oscillations and transmission capacity. This model does not include detailed representations of the power electronics, the measurement system, or the synchronization system. These systems are approximated rather by simple transfer functions that yield a correct representation at the system's fundamental frequency.

1 tcr3tsc.

A detailed model of a SVC using three TSCs and one TCR is provided in the power svc

The SVC can be operated in two different modes:

- In voltage regulation mode (the voltage is regulated within limits as explained below)

- In var control mode (the SVC susceptance is kept constant)

When the SVC is operated in voltage regulation mode, it implements the following V-I characteristic.

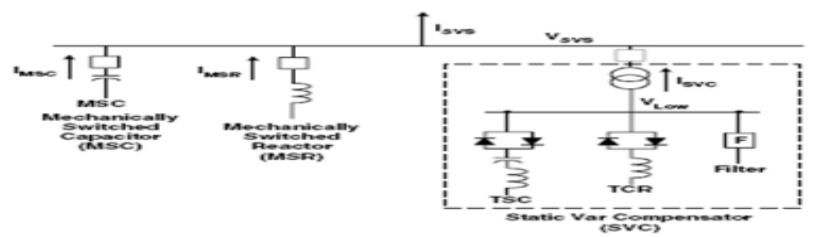

Fig 2 Circuit for a Static Var Compensator

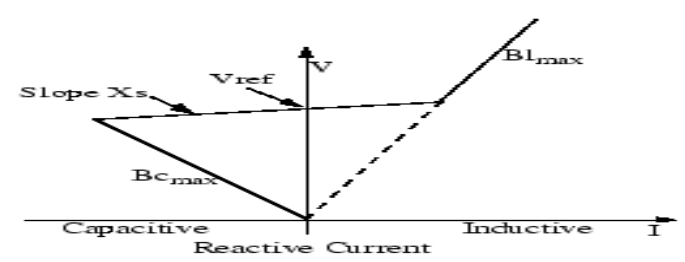

Fig 3. SVC V-I characteristic

As long as the SVC susceptance B stays within the maximum and minimum susceptance values imposed by the total reactive power of capacitor banks $\left(\mathrm{B}_{\mathrm{cmax}}\right)$ and reactor banks $\left(\mathrm{B}_{\operatorname{lmax}}\right)$, the voltage is regulated at the reference voltage $\mathrm{V}_{\text {ref. }}$. However, a voltage droop is normally used (usually between $1 \%$ and $4 \%$ at maximum reactive power output), and the V-I characteristic has the slope indicated in the figure. The V-I characteristic is described by the following three equations:

$V=V_{\text {ref }}+X_{s} . I \quad \mathrm{SVC}$ is in regulation range $\left(-B_{\max }<B<B l_{\max }\right)(3.1 .1)$ 


$$
\begin{array}{cc}
V=-\frac{I}{B c_{\max }} & \text { SVC is fully capacitive }\left(B=B c_{\text {max }}\right) \\
V=-\frac{I}{B l_{\max }} & \text { SVC is fully inductive }\left(B=B l_{\max }\right)
\end{array}
$$

Where

V Positive sequence voltage $(\mathrm{pu})$

I Reactive current (pu/Pbase) (I >0 indicates an inductive current)

$\mathrm{X}_{\mathrm{s}}$ Slope or droop reactance (pu/Pbase)

$\mathrm{B}_{\mathrm{cmax}}$ Maximum capacitive susceptance (pu/Pbase) with all TSCs in service, no TSR or TCR

$\mathrm{B}_{\text {Imax }}$ Maximum inductive susceptance (pu/Pbase) with all TSRs in service or TCRs at full conduction, no TSC Pbase Three-phase base power specified in the block

\section{A.1 Modeling and Control of SVC}

The Static Var Compensator is basically a shunt connected variable Var generator whose output is adjusted to exchange capacitive or inductive current to the system. One of the most widely used configurations of the SVC is the FC- TCR type in which a Fixed Capacitor (FC) is connected in parallel with Thyristor Controlled Reactor (TCR). The magnitude of the SVC is inductive admittance BL $(\alpha)$ is a function of the firing angle $\alpha$ and is given by.

$$
B_{L}(\alpha)=\frac{2 \pi-2 \alpha+\sin 2 \alpha}{\pi X_{S}}
$$

For $\pi / 2 \leq \alpha \leq \pi$ where $X_{S}=\frac{V_{S}^{2}}{Q_{L}}$

$\mathrm{Vs}=\mathrm{SVC}$ bus bar voltage and $\mathrm{QL}=\mathrm{MVA}$ rating of reactor. As the SVC uses a fixed capacitor and variable reactor combination (TCR- FC), the effective shunt admittance is

$$
B_{S}=\frac{1}{X_{c}}-B_{L}(\alpha)
$$

Where $\mathrm{X}_{\mathrm{c}}=$ Capacitive reactance.

An SVC with firing control system can be represented, for the sake of simplicity by a first order model characterized by a gain KSVC and time constants T1 and T2 as shown in Fig.1 The controller send firing control signals to the thyristor switching unit to modify the equivalent capacitance of the SVC. The fuzzy controller provides a auxiliary control, which is in addition to the voltage feedback loop.

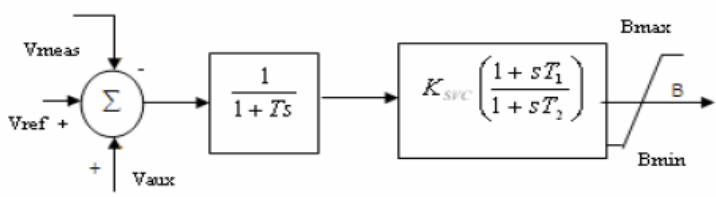

Fig A.1 Block Representation of SVC Control

The auxiliary control loop of the SVC uses stabilizing signals, such as speed, frequency, phase angle difference etc.,to improve the dynamic performance of the system.

This figure shows that the speed of mechanical switches (primarily circuit breakers) for conventional equipment solutions can be as fast as a couple of cycles of 60(or 50) Hz. This speed of switching in and of itself may be fast enough to solve many power system constraints. Although there is a vast improvement in switching time from mechanical to power electronic based solutions (Figure 3) illustrates that the speed of power electronics switches is a fraction of a cycle), the main benefit that FACTS controller solutions provide is the "cycling/repeatability" and "smooth control" that accompanies the power electronic based switching. In other words, a mechanically switched based (conventional) solution is usually a "one and done" or "on or off" impact to the power system in the time frame needed for power system stability, whereas the power electronic based solution can provide a smooth, continuous, and/or repeatable option for power system control. Thus by applying power electronic based solutions to alleviate power system constraints, it is not just "speed" but "cycling" and "smooth control" that is gained.

\section{A.2 Benefits of Control of Power Systems}

Once power system constraints are identified and through system studies viable solutions options are identified, the benefits of the added power system control must be determined. The following offers a list of such benefits:

Increased Loading and More Effective Use of Transmission Corridors

- Added Power Flow Control

- Improved Power System Stability 
- Increased System Security

- Increased System Reliability

- $\quad$ Added Flexibility in Siting New Generation

- Elimination or Deferral of the Need for New Transmission Lines

Such benefits can usually be tied back to an area or region for a specific season and year at a defined dispatch (usually given by an ISO or equivalent) while meeting the following criteria, for example:

- Voltage Stability Criteria

- Dynamic Voltage Criteria

- Transient Stability Criteria

\section{B FUZZY LOGIC CONTROLLERS \\ B.1Fuzzy Inference system}

With cause effect relationship expressed as a collection of fuzzy if - then rules, in which the preconditions uses linguistic variables and the consequent have class labels, qualitative reasoning is performed to infer the results. In our model Mamdani inference system with product t-norm and max t-co norm is used. Here, the set of sensor input is matched against the if part of each if - then rule, and the response of each rule is obtained through fuzzy implication operation. The response of each rule is weighted according to the extent to which each rule fires. The response of all the fuzzy rules for a particular output class are combined to obtain the confidence with which the sensor input is classified to that fault class.

B.1 Power quality improvement of power generation using FLC based Damping Controller design Fig.2 shows the schematic diagram of a SVC along with Fuzzy logic based damping controller. Generator speed deviation $(\Delta \omega)$ and $(\Delta \mathrm{P})$ are taken as the input signals of the fuzzy controller

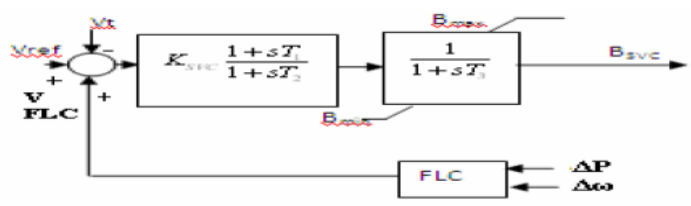

Fig. 6.1 Block of proposed Fuzzy logic controller diagram

The number of membership functions for each variable determines the quality of control which can be achieved using fuzzy logic controllers. In the present investigation, five membership functions are defined for the input and output variables. Fig.3 shows the membership functions defined. The mentioned membership functions are used to specify a set of rules called a rule base. With two inputs and five linguistic terms, 25 rules were developed which is given in Table 1. In inference mechanism all the rules are compared to the inputs to determine which rules apply to the current situation. After the matching process the required rules are fired. The controlled output $\mathrm{B}_{\mathrm{svc}}$ is determined for the different input conditions. The de-fuzzification produces the final crisp output of FLC with the fuzzified input. Centroid method is employed where the output will be calculated as

$$
O / P=\frac{\sum_{i=1}^{5} b_{i} \int \mu_{(i)}}{\sum_{i=1}^{5} \int \mu_{(i)}}
$$

Fig b.1.1 Membership functions of $\Delta \omega, \Delta \mathrm{P}$ and $\mathrm{B}_{\mathrm{svc}}$

\begin{tabular}{|c|c|c|c|c|c|c|}
\hline \multirow{2}{*}{\multicolumn{2}{|c|}{$\begin{array}{l}\text { Output } \\
\text { (B svc) }\end{array}$}} & \multicolumn{5}{|c|}{ FUZZY INFERENCE RULES } \\
\hline & & & & $\Delta P$ & & \\
\hline \multirow{6}{*}{$\Delta \omega$} & & NB & NS & $z$ & PS & $\mathrm{PB}$ \\
\hline & $N B$ & NB & NS & NB & NS & $Z$ \\
\hline & NS & NS & NB & $z$ & $z$ & PS \\
\hline & Z & NS & $z$ & $z$ & PS & PS \\
\hline & PS & Z & Z & PS & PS & PS \\
\hline & PB & Z & PS & PS & PS & $\mathrm{PB}$ \\
\hline
\end{tabular}

Fig 6.1.2 Fuzzy Inference Rules Fuzzy Inference Rules 


\section{B.2Modeling of Power System Components \\ B.1. Generator}

The generator is represented by third order model comprising the electromechanical swing equation and the generator internal voltage equations [10] are

$$
\begin{aligned}
& \dot{\omega}=\frac{1}{M}\left(\mathbf{P}_{\mathrm{m}}+\mathbf{G}+\mathbf{K}_{\mathrm{d}} \boldsymbol{\omega}-\mathbf{P}_{\mathrm{e}}\right) \quad \text { b.1.1 } \\
& \dot{E}_{q}^{\prime}=\frac{1}{T_{d o}{ }^{\prime}}\left[E_{f d}-\left(X_{d}-X_{d}^{\prime}\right) i_{d}-E_{q}^{\prime}\right]
\end{aligned}
$$

$$
\dot{\delta}=\omega_{0} \omega
$$

Where $\delta=$ Rotor angle in degrees

$\omega=$ Angular speed in $\mathrm{rad} / \mathrm{sec}$

$\mathrm{P}_{\mathrm{m}}=$ Mechanical power developed by the generator

$\mathrm{K}_{\mathrm{d}}=$ Damping constant of the generator

$\mathrm{P}_{\mathrm{e}}=$ Electrical Power delivered in p.u

$X_{d}, X_{q}=$ Direct and quadrature axis reactance of the generator in p.u

$E_{d}, E_{q}=$ Direct and quadrature axis voltages behind the transient reactance in p.u

\section{B.2 Power quality improvement of distribution by minimization of harmonics by FLC based sve control}

The PQ at the PCC is expressed in terms of various parameters. Total harmonic distortion (THD) or total demand distortion (TDD) at PCC is one of these

parameters, which is commonly used in practice. If = fundamental current;

$\mathrm{I}_{\mathrm{d}}=$ demand current;

$\mathrm{I}_{\mathrm{h}}=$ harmonic line current;

$\mathrm{m}=$ maximum order of harmonics considered.

Assuming balanced three-phase voltages at the

load bus, the fundamental and harmonic components of

the line currents can be obtained by using the following

By setting balanced values for QC and QS, the unbalanced reactive power absorptions of TCR can be obtained by using the procedure described above. Now, the unsymmetrical reactance required absorbing $\mathrm{QR}$ and the corresponding Unsymmetrical firing angles can be computed from Eqn28. Knowing the voltages at the compensator node and the firing angles of the TCR, harmonic analysis can

be carried out and the performance index THD can be evaluated as explained. Thukaram et al. have shown in [11] that different combinations of firing angles lead to various harmonic levels, as indicated by the value of the performance index. In order to minimize the harmonics generated due to SVC operation, the TCR should be operated at a combination of firing angles which results in a low harmonic level. It has been further shown that there are several combinations of firing angles which lead to a lower level of harmonic generation. The combination of firing angles that corresponds to the minimum THD value usually conflicts with the objective of minimizing the reactive power drawn from the source. Therefore, it is necessary to find a combination of firing angles, which can simultaneously keep QS and THD satisfactorily low. However, the task of selecting the particular combination firing angles from a set of all (or many) plausible combinations of firing angles to achieve optimum values of QS and THD is not straightforward. In this paper, fuzzy logic and the ANN controller are used to obtain the triggering delay angles $\alpha 1, \alpha 2$, and $\alpha 3$ for the TCR. These triggering delay angles correspond to minimum THD values and acceptable compromised reactive power QS.

\section{B2.1 SVC Control With Fuzzy Ranking System}

It is reported in the literature that Mamdani-type fuzzy controllers give better performance for reactive power control of TSC-TCR circuits. A Mamdani-type fuzzylogic system was designed for ranking the combinations of TSC step size and three firing angles. The schematic diagram of the SVC control algorithm, shown in Fig. takes phase-wise active and reactive power demands of

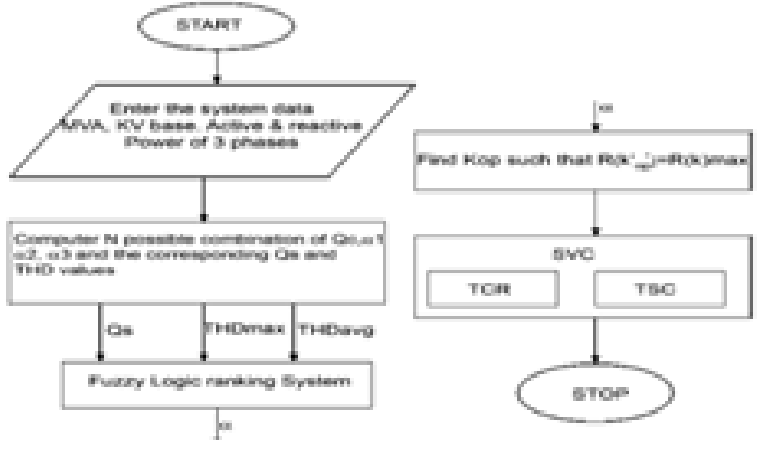


the load as inputs and determines the step size of TSC and the unsymmetrica1 firing angles of the TCR as outputs. The first block computes a set of feasible

combinations (say, $\mathrm{N}$ different combinations) of firing angles $\alpha 1, \alpha 2, \alpha 3$ and the corresponding QS and THD values. The second block is the ranking of each feasible

TSC step size-firing angles combination using the fuzzy-logic ranking system. The fuzzy-logic ranking system assigns a ranking score, $\mathrm{R}(\mathrm{K})$ for the $\mathrm{N}$ th combination depending on the corresponding $\mathrm{QS}(\mathrm{K})$ and $\mathrm{THD}(\mathrm{K})$ values. In the case of three-phase unbalanced loads, three different THD values

\section{SIMULATION RESULTS}

To assess the effectiveness of the proposed controller, simulation studies are carried out for the most severe fault conditions and overload conditions in both SMIB system and Multi machine system. The details of the simulation are presented here. 7.1. SMIB system for generation and transmission with svc A SMIB system, equipped with Generator, Transmission line and SVC at the midpoint of the line is shown in fig.7.1 The SVC with its controller is place at the midpoint of the transmission line. The fuzzy damping controller for the SVC is developed using MATLAB / SIMULINK and its block diagram is shown in fig. A three phase fault is simulated at the load end at $\mathrm{t}=0.1 \mathrm{sec}$. and cleared after $0.05 \mathrm{sec}$. The system response without SVC is oscillatory and leads to instability.

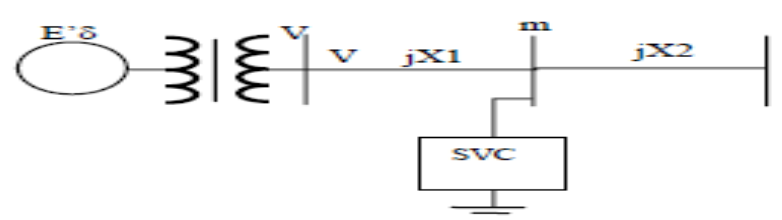

Fig3.1 Single Line Diagram of SMIB System

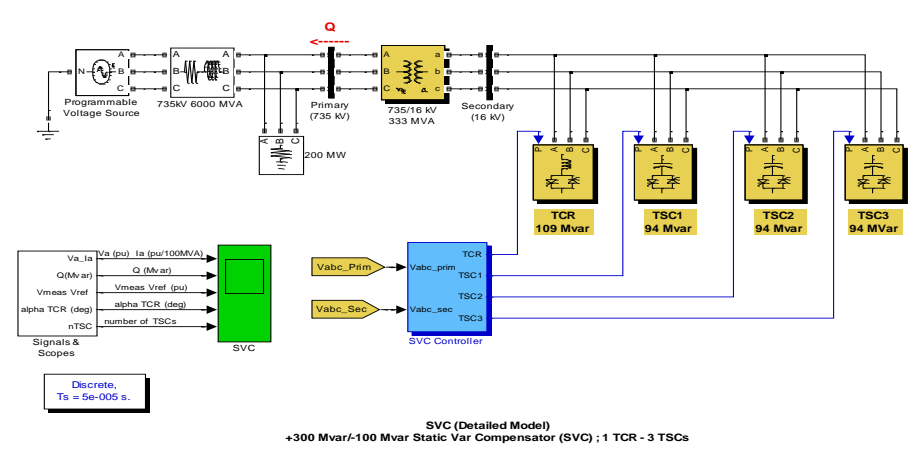

Fig 3.2 Static Var Compensator With Controller

\section{Variation in rotor angle for different controllers of SVC}

Variation in rotor angle with Fuzzy controller:

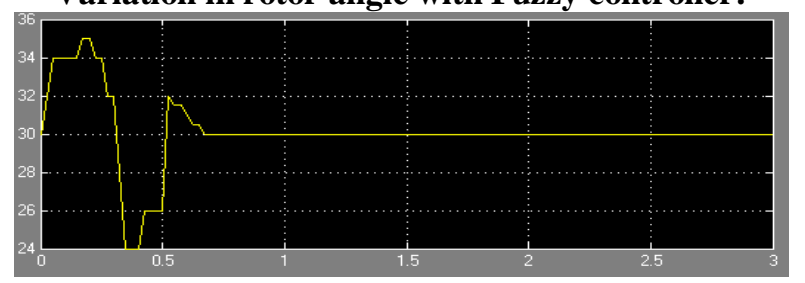

Variation in speed deviation with Fuzzy controller:

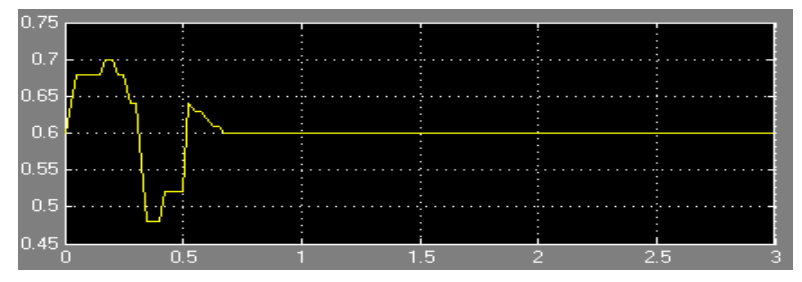


The SVC voltage increases during fault period which causes additional voltage injection in the system instead of current injection. This will be the remarkable advantage while using a FLC based controller.

\section{Damping of rotor angle oscillations for loading condition of 1.5 p.u:}

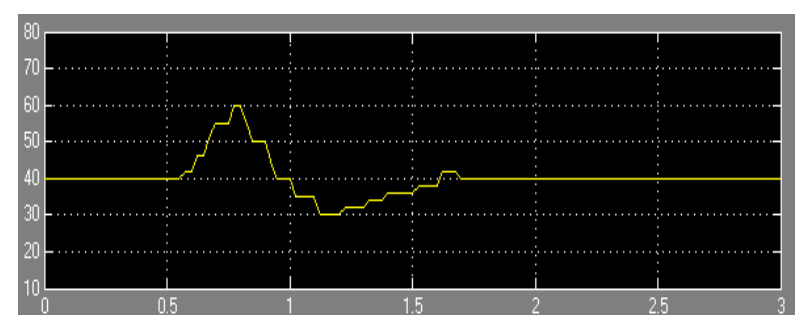

From the Fig. it can be seen that, complete damping of rotor angle oscillations occur with FLC controller damp out the oscillations at 1.5 seconds.

\section{SMIB system for distribution with sve}

An 11-kV/400-V, 200-kVA distribution substation feeding a fluctuating load is taken for simulation as shown in Fig. 2. The load consists of single-phase and three-phase motors, laboratory equipment, and switchedmode power supplies.

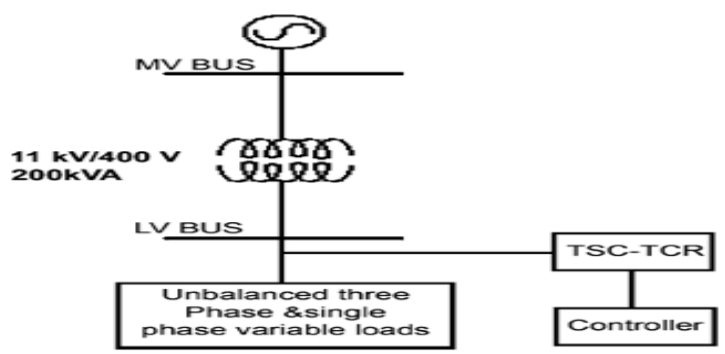

Fig 3,3 oneline diagram distribution system with svc

The static VAR compensator was considered consisting of a TSC that can vary through four steps; 0 , 10,20 , and $30 \mathrm{kVAR}$ per phase and a thyristor-controlled reactor (TCR) of capacity of $10 \mathrm{kVAR}$ per phase under full conduction.

The simulated results using ANN in the MATLAB 7.0 environment for ten samples at two seconds each are shown in table below

\begin{tabular}{|c|c|c|c|c|c|}
\hline \multirow{2}{*}{ 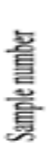 } & \multicolumn{3}{|c|}{ Load $(P+j Q) k V A$} & \multicolumn{2}{|c|}{$\%$ THDDrs } \\
\hline & $\mathbf{R}$ & Y & B & 产 & 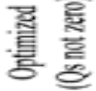 \\
\hline 1 & $14+j 26$ & $27+j 5$ & $30+j 15$ & 18.65 & 8.70 \\
\hline 2 & $24+j 15$ & $17+j 28$ & $15+j 26$ & 37.29 & 13.13 \\
\hline 3 & $13+j 22$ & $17+j 26$ & $17+j 24$ & 16.15 & 8.30 \\
\hline 4 & $20+j 12$ & $25+j 13$ & $30+j 15$ & 8.74 & 5.41 \\
\hline 5 & $25+j 25$ & $25+j 25$ & $25+j 25$ & 13.35 & 11.53 \\
\hline 6 & $10+j 05$ & $15+j 22$ & $25+j 12$ & 2.55 & 1.47 \\
\hline 7 & $14+j 25$ & $15+j 15$ & $10+j 25$ & 13.55 & 6.96 \\
\hline 8 & $12+j 15$ & $30+j 29$ & $17+j 21$ & 24.41 & 12.79 \\
\hline 9 & $14+j 26$ & $12+j 15$ & $19+j 26$ & 22.39 & 11.01 \\
\hline 10 & $19+j 22$ & $30+j 18$ & $19+j 21$ & 15.66 & 8.70 \\
\hline
\end{tabular}

fig 3.4 average values of THD at different load

For each load data, Avg. shows the reactive power drawn from the source for optimized. The percentage average THD for unoptimized $(Q=0)$ operation shows the percentage average THD when SVC perfectly balances the reactive power whereas for optimized ( $Q$ not zero) operation indicates the percentage average 


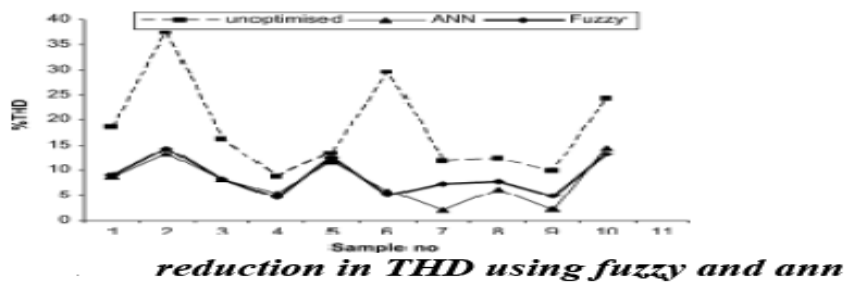

compared to operation, clearly showing that the ANN controller follows the trend. The THD profile of one of the phases using the ANN controller

\section{CONCLUSION}

This paper presents the application of a fuzzy logic based auxiliary control for an SVC to achieve transient stability enhancement. ideal solution, suffers from serious limitations of high cost, high losses, and complex control strategy. The SVCs, while correcting power factor, inject harmonics in distribution lines. The operation of thyristor controlled compensators (TSC-TCR) at various conduction angles can be used to advantageously meet the unbalanced reactive power demands in a fluctuating load environment. The proposed ANN-based approach can be effectively used to reduce and balance the reactive power drawn from the source under unbalanced loadings while keeping the harmonic injection into the power system low. The proposed FLC for SVC is proved to be very effective and robust in damping power system oscillations and thereby enhancing system transient stability. Fuzzy rules are easily derived from the measurable global signals like line active power flow, and remote generator speed deviation. The performance of various controllers is then compared based on non linear simulation results. Among these the performance of the proposed controller is found to be better and damp out the system oscillations at faster rate. It was also observed power quality improvement of fuzzy logic control of svc for generation transmission and distribution network of power system. The SMIB system. Digital computer simulations were performed using MATLAB/ SIMULINK software.

\section{Acknowledgment}

The authors would like to thank the authorities of Ghousia College of Engineering, Ramanagaram, Karnataka, India for all the cooperation and encouragement in carrying out this work.

\section{References}

[1] S. Mohagheghi, Student Member, IEEE, G.K. Venayagamoorthy, Senior Member, IEEE, R.G. Harley,Mamdani based fuzzy logic controller for static compensator in a multi machine system Fellow, IEEE TRANSACTIONS ON POWER SYSTEMS, VOL. 21 , NO. 4, NOVEMBER 20061

[2] D.Harikrishna, R.S.Dhekekar, *N.V.Srikanth,,A novel approach to small signal stability enhancement using fuzzy logic controller of SVC,IEEE Transactions on Power Systems, Vol. 15, No. 4, November 2000, pp 1360-1366

[3] Takashi Hiyama, Senior Member ECE Department ECE Department ECE Department Kumamoto University, Walid Hubbi, Senior Member Thomas H., Fuzzy logic control scheme with variable for SVC to enhance powersystem stability ,CLEI ELECTRONIC JOURNAL, VOLUME 14, NUMBER 1, PAPER 5, APRIL 2011

[4] D. Z. Fang, Yang Xiaodong, T. S. Chung, Senior Member,Adaptive Fuzzy-Logic SVC Damping Controller Using Strategy of Oscillation Energy Descent IEEE, and K. P. Wong, Fellow, IEEE IEEE TRANSACTIONS ON POWER SYSTEMS, VOL. 19, NO. 3, AUGUST 2004

[5] Salman.Mohagheghi, Student Member, IEEE, Ganesh kumar. Venayagamoorthy, Senior Member, IEEE, Novel nonlinear optimal controller for static compensator, IEEE Transactions on Power Systems, Vol. 19 , Issue 1 , February 2005,IEEE Transactions on Power Systems, Vol. 19 , Issue 1, February 2004

[6] Prechanon Kumkratug Department of Electrical Engineering, Faculty of Engineering at Si Racha, Kasetsart University, 199 M. 6, Tungsukhla, Si Racha, Chonburi, 20230, Thailand ,Static Var Compensator based on Fuzzy Logic Control for Damping Power System OscillationAmerican Journal of Applied Sciences 8 (10): 1027-1031, 2011 ISSN 1546-9239@ 2011 Science Publications

[7] M.H.Hemmatpour,M.Mohammadian, Optimum reconfiguration with FACTS devices in distribution system,,IEEE Transactions on Power Delivery, Vol. 12, No. 4, . 1635-1641, 1997.

[8] M. Rajaram Vice Chancellor, Anna University of Technology Power System Damping Using Neuro-Fuzzy Based Static Synchronous Series Compensator Controllers European Journal of Scientific Research ISSN 1450-216X Vol.60 No.1 (2011), pp. 149-158@ EuroJournals Publishing, Inc. 2011

[9] P.K. Dash S. Mishra Design of a robust controller for the auxillary control loop of a static var compensator A.C. Liew ,IEEE Transm Distrib, Val 142, No 6, November 1995

[10] N.Karpagam,D.Devaraj, Fuzzy Logic Control of Static Var Compensator for Power System Damping ,International Journal of Electrical Power and Energy Systems Engineering 2:2 2009 